\title{
INCREASING CROPPING INTENSITY AND CROP PRODUCTIVITY OF FOUR CROPS BASED MUSTARD-BORO-T. AUS-T. AMAN CROPPING PATTERN
}

\author{
A. Barman ${ }^{1}$, S. Shome ${ }^{2}$, M.R. Khatun ${ }^{1}$, M.M. Masud ${ }^{1}$ and S. Akther ${ }^{1}$ \\ ${ }^{1}$ Soil Science Division, BARI, Gazipur, Bangladesh \\ ${ }^{2}$ Department of Agronomy, SAU, Dhaka, Bangladesh \\ Corresponding E-mail: alakbarman.sau@gmail.com
}

(Received: 19 April 2021, Accepted: 01 May 2021)

Keywords: Cropping system; fertilizer; marginal benefit cost ratio; rice equivalent yield; yield

\begin{abstract}
A field trial on soil test based (STB) fertilizer doses was conducted during the year of 2017-2018 and 2018-2019 in Jashore region (AEZ-11) to find out the most suitable fertilizer doses for four crop based cropping pattern considering the agronomic feasibility and economic return of the system. The experiment consisted of eight different treatments viz. $\mathrm{T}_{1}: 100 \% \mathrm{NPKSZnB}$ (STB), $\mathrm{T}_{2}: \mathrm{T}_{1}+25 \% \mathrm{~N}, \mathrm{~T}_{3}$ : $\mathrm{T}_{1}+25 \% \mathrm{NP}, \mathrm{T}_{4}: \mathrm{T}_{1}+25 \% \mathrm{NK}, \mathrm{T}_{5}: \mathrm{T}_{1}+25 \% \mathrm{PK}, \mathrm{T}_{6}: \mathrm{T}_{1}+25 \% \mathrm{NPK}, \mathrm{T}_{7}: 75 \%$ of $\mathrm{T}_{1}, \mathrm{~T}_{8}$ : Native fertility. Randomized complete block design (RCBD) with three replications was followed. Data revealed that seed yield of mustard was remarkably influenced by fertilizer treatments while grain yield of other components of the cropping system was not affected significantly by the treatments except control or native fertility. It was observed that $25 \%$ more NPK over $100 \%$ STB dose provided the highest yield of all the component crops. The highest rice equivalent yield (3.34 $\left.t \mathrm{ha}^{-1}\right)$ was recorded from $\mathrm{T}_{6}$ and the lowest $\left(1.88 \mathrm{t} \mathrm{ha}^{-1}\right)$ from $\mathrm{T}_{8}$ treatment. Maximum gross return (Tk. 420000/ha) and marginal benefit cost ratio (4.08) were also obtained from $\mathrm{T}_{6}$ treatment. So, $25 \% \mathrm{NPK}+100 \%$ STB dose of fertilizer could be followed for productive and remunerative rice based cropping system MustardBoro-T. Aus-T.Aman in AEZ-11.
\end{abstract}

\section{Introduction}

Bangladesh is one of the highest populous countries of the world with the annual growth rate of about 1.37\% (BBS, 2019). On the other hand, available agricultural land of Bangladesh is decreasing with an alarming rate of about $1 \%$ per year (Hossain et al., 2014). Rate of cropland shifting to non-agricultural land (housing, industry, etc.) is formidable as it is associated with the food security of the country (Islam et al., 2018). Meeting the challenge of ensuring food security through horizontal expansion of land is not possible due to decrease in agricultural land. So intensifying land use system through multiple cropping or by growing more and more crops on the same piece of land in a calendar year is a promising option to feed this teeming millions.

Rice based cropping system consisting of Boro-Fallow-T. Aman is a popular cropping pattern of Bangladesh (Parvin et al., 2017). Inclusion of Mustard and short duration T. Aus variety in this cropping pattern could increase the cropping intensity of our country up to $400 \%$ as well as improve the socio-economic condition of the farmer. However, the advantage of including extra crops in rice based cropping systemcould depends upon the selection of variety and appropriate agronomic management practices such as fertilizer management (Hossain et al., 2014). Aziz et 
al. (2013) reported that multiple cropping system effects soil carbon and nitrogen status, and also improve soil functional properties. Nevertheless, continuous cropping causes nutrient mining from soil while a blanket fertilizer dose for all regions without considering the soil nutrient status leads to tremendous damage to soil, environment and economy. Sultana et al. (2015) opined that actual recommended fertilizer dose is higher than actual need of fertilizer and this gap creates soil nutrient imbalance.As soil fertility is a major determinant for the success and failure of a crop production system, time demands a suitable fertilizer recommendation based on soil testing for promising four crops based cropping pattern which is agronomically feasible and economically profitable. As such, the present study was undertaken to find out the most suitable fertilizer dose for Mustard-Boro-T.Aus-T.Aman cropping pattern at AEZ-11 considering both the yield and economic return of the system.

\section{Materials and Methods}

\section{Initial soil status}

The field experiment was executed at the central farm of RARS, Jashore (AEZ 11) during 201718 and 2018-19. The initial soil samples from 0-15 cm depth was collected and analyzed before establishing the experiment. Initial soil status of experimental field is presented in Table 1.

Table 1. Chemical properties of experimentalsoil (initial) at the RARS, Jashore

\begin{tabular}{|c|c|c|c|c|c|c|c|c|c|c|c|c|}
\hline \multirow[t]{2}{*}{$\mathrm{pH}$} & \multirow{2}{*}{$\begin{array}{l}\text { OM } \\
(\%)\end{array}$} & $\mathrm{Ca}$ & $\mathrm{Mg}$ & $\mathrm{K}$ & \multirow[t]{2}{*}{ Total N \% } & $P$ & $\mathrm{~S}$ & $\mathrm{~B}$ & $\mathrm{Cu}$ & $\mathrm{Fe}$ & Mn & Zn \\
\hline & & $\mathrm{me}$ & 100 & 3 soil & & \multicolumn{7}{|c|}{$\mu \mathrm{g} \mathrm{g}^{-1}$} \\
\hline 7.7 & 0.92 & 12 & 3.4 & 0.19 & 0.048 & 15.1 & 18.0 & 0.14 & 2.1 & 38 & 5.0 & 2.0 \\
\hline Crit & al level & 2.0 & 0.5 & 0.12 & Very low & 7.0 & 10 & 0.2 & 0.2 & 4.0 & 1.0 & 0.6 \\
\hline
\end{tabular}

\section{Experimental details}

The experiment was conducted in a Randomized complete block design with three replications. Eight different treatments viz. $\mathrm{T}_{1}: 100 \% \mathrm{NPKSZnB}$ (STB), $\mathrm{T}_{2}: \mathrm{T}_{1}+25 \% \mathrm{~N}, \mathrm{~T}_{3}: \mathrm{T}_{1}+25 \% \mathrm{NP}$, $\mathrm{T}_{4}: \mathrm{T}_{1}+25 \% \mathrm{NK}, \mathrm{T}_{5}: \mathrm{T}_{1}+25 \% \mathrm{PK}, \mathrm{T}_{6}: \mathrm{T}_{1}+25 \% \mathrm{NPK}, \mathrm{T}_{7}: 75 \%$ of $\mathrm{T}_{1}, \mathrm{~T}_{8}$ : Native fertility was applied in different plots having size of $4.2 \mathrm{~m} \times 3 \mathrm{~m}$ each. The STB fertilizer dose for mustard, boro, T.Aus and T.Aman is $\mathrm{N}_{138} \mathrm{P}_{22} \mathrm{~K}_{25} \mathrm{~S}_{0} \mathrm{Zn}_{1.85}, \mathrm{~N}_{186} \mathrm{P}_{30} \mathrm{~K}_{23} \mathrm{~S}_{30} \mathrm{Zn}_{0}, \mathrm{~N}_{90} \mathrm{P}_{15} \mathrm{~K}_{2.17} \mathrm{~S}_{23} \mathrm{Zn}_{0}$ and $\mathrm{N}_{159} \mathrm{P}_{30} \mathrm{~K}_{7} \mathrm{~S}_{24} \mathrm{Zn}_{0}$, respectively. Mustard crop (var. BARI Sarisha-14) was used as test crop for the first component of the pattern. Mustard seeds were sown in line with $30 \mathrm{~cm}$ row to row on November 06, 2017 and November 07, 2018. Fertilizer N-P-K-S-Zn and B were supplied from urea, TSP, MP, Gypsum, Zinc sulphate and Boric acid respectively. All PKSZnB and 1/3 of N were applied at the time of final land preparation. The remaining two third of $\mathrm{N}$ were applied as top dress at 30 and 60 days after sowing. Three irrigation and other intercultural operations were done as and when required. The mustard was harvested on January 28, 2018 and January 27, 2019. After mustard, BRRI dhan28 was transplanted in the same plots on February 3, 2018 and February 2, 2019 with a row to row spacing of $20 \mathrm{~cm}$ and plant to plant spacing of $15 \mathrm{~cm}$. All fertilizers including $1 / 3^{\text {rd }}$ of $\mathrm{N}$ were applied before transplanting. Rest of $\mathrm{N}$ was applied in two installments at 15 and 45 days after transplanting. For T. Aus rice, the variety BRRI dhan48 was used in the experiment. Transplanting was done on May 15, 2018 and May 14,2019 with a row to row spacing of $20 \mathrm{~cm}$ and plant to plant spacing of $15 \mathrm{~cm}$. All fertilizers including $1 / 3^{\text {rd }}$ of $\mathrm{N}$ were applied before transplanting. Rest of $\mathrm{N}$ was applied in two installments at 15 and 45 days after transplanting. For T. Aman rice, the variety BRRI dhan57 was used in the experiments. Transplanting was done on July 29, 2018 and July 28, 2019 with 
a row to row spacing of $20 \mathrm{~cm}$ and plant to plant spacing of $15 \mathrm{~cm}$. T. Aman rice was harvested on November 4, 2018 and November 5, 2019.

\section{Data collection and analysis}

Data on yield and yield contributing characters were recorded and analyzed using Statistix 10 software. The means were separated using least significant difference (LSD) at the 5\% level of significance. Rice equivalent yield is determined by the following equation

Rice equivalent yield of a crop $=\frac{\text { Yield of that crop }\left(\mathrm{t} \mathrm{ha}^{-1}\right) \times \text { unit price of that crop }}{\text { Unit price of rice }}$

\section{Economic analysis}

Gross return from this rice based cropping system was calculated by multiplying the yield of crops with their market price. Total variable cost included the fertilizer cost. Gross margin was calculated by subtracting the variable cost from the gross return. Marginal Benefit Cost Ratio (MBCR) was calculated by dividing the marginal value product by total variable cost.

\section{Results and Discussion}

\section{Mustard}

Data regarding yield and yield contributing characters of mustard as influenced by the fertilizer rate has been presented in Table 2 \& 3. In both the experimental years (2017-2018 and 20182019), $25 \%$ NPK $+100 \%$ STB gave highest plant height, no. of branches plant ${ }^{-1}$, no. of pods plant $^{-1}$, no. of grains pod $^{-1}, 1000$ seed weight (g) and stover yield while no fertilizer gave the lowest value of these parameters. In the two consecutive years, about $46 \%$ and $48 \%$ more yield was obtained than 100\% STB dose while 25\% more NPK was added to 100\% STB dose. The native nutrient treatment produced the lowest mustard seed yield $(0.91$ and 0.96$) \mathrm{t} \mathrm{ha}^{-1}$ in Jashore during the year of 2017-18 and 2018-19. Alike trend of increased yield in mustard due to added fertilizers in 3 or 4 cropping patterns was also reported by Barman et al. (2019) and Saha et al. (2016). Dobermann et al. (2013) opined that site-specific plant nutrient management (STB in our study) can increase yield as well as nutrient use efficiency of crop rotation.

Table 2. Yield and yield contributing characters of Mustard during 2017-2018

\begin{tabular}{|c|c|c|c|c|c|c|c|c|c|}
\hline \multirow{2}{*}{$\begin{array}{l}\text { Treat } \\
\text { ment }\end{array}$} & \multirow{2}{*}{$\begin{array}{l}\text { Plant } \\
\text { height } \\
\text { (cm) }\end{array}$} & \multirow{2}{*}{$\begin{array}{c}\text { No. of } \\
\text { branches }\end{array}$} & \multirow{2}{*}{$\begin{array}{l}\text { No. of } \\
\text { pods } \\
\text { plant }^{-1}\end{array}$} & \multirow{2}{*}{$\begin{array}{l}\text { Pod } \\
\text { length } \\
\text { (cm) }\end{array}$} & \multirow{2}{*}{$\begin{array}{l}\text { No. of } \\
\text { grains } \\
\text { pod }^{-1}\end{array}$} & \multirow{2}{*}{$\begin{array}{c}\text { Ineffective } \\
\text { pods } \\
\text { plant }^{-1}\end{array}$} & \multirow{2}{*}{$\begin{array}{c}1000- \\
\text { seed } \\
\text { weight (g) }\end{array}$} & $\begin{array}{l}\text { Seed } \\
\text { yield }\end{array}$ & $\begin{array}{c}\text { Stover } \\
\text { yield }\end{array}$ \\
\hline & & & & & & & & \multicolumn{2}{|c|}{ (t ha-1) } \\
\hline $\mathrm{T}_{1}$ & $82.03 b$ & 5.53de & 63.53de & $4.00 b c$ & $32.53 c$ & 9.33bcd & $3.40 \mathrm{~cd}$ & $1.10 \mathrm{c}$ & $2.26 b$ \\
\hline $\mathrm{T}_{2}$ & $84.67 \mathrm{ab}$ & $6.73 b c$ & $73.53 c$ & $4.40 \mathrm{ab}$ & $35.46 \mathrm{abc}$ & $11.00 \mathrm{abc}$ & $3.66 b c$ & $1.12 \mathrm{c}$ & $2.30 \mathrm{~b}$ \\
\hline $\mathrm{T}_{3}$ & $87.60 \mathrm{a}$ & $7.00 \mathrm{~b}$ & $87.46 b$ & 4.53ab & $36.80 \mathrm{ab}$ & $9.00 \mathrm{~cd}$ & $3.83 b$ & $1.43 b$ & $2.43 \mathrm{ab}$ \\
\hline $\mathrm{T}_{4}$ & 85.86ab & $6.86 b c$ & $86.33 b$ & 4.53ab & $35.53 \mathrm{abc}$ & $8.00 \mathrm{~d}$ & $3.70 b c$ & $1.23 \mathrm{c}$ & $2.36 \mathrm{~b}$ \\
\hline$T_{5}$ & $82.80 \mathrm{~b}$ & $5.80 \mathrm{~cd}$ & $67.00 \mathrm{~cd}$ & $4.40 \mathrm{ab}$ & $34.40 b c$ & 11.66ab & $3.46 \mathrm{bcd}$ & $1.21 \mathrm{c}$ & $2.30 \mathrm{~b}$ \\
\hline $\mathrm{T}_{6}$ & $89.33 a$ & $8.46 a$ & $96.20 \mathrm{a}$ & $4.93 a$ & $38.40 \mathrm{a}$ & $7.00 \mathrm{~d}$ & $4.26 \mathrm{a}$ & $1.61 \mathrm{a}$ & $2.70 \mathrm{a}$ \\
\hline $\mathrm{T}_{7}$ & $75.66 c$ & $5.40 \mathrm{de}$ & $59.00 \mathrm{ef}$ & $3.93 b c$ & $28.53 d$ & $12.66 a$ & $3.20 \mathrm{de}$ & $1.01 \mathrm{~d}$ & $2.16 \mathrm{~b}$ \\
\hline $\mathrm{T}_{8}$ & $61.86 \mathrm{~d}$ & $4.53 e$ & $55.33 \mathrm{f}$ & $3.60 c$ & $25.00 e$ & $13.33 a$ & $2.96 e$ & $0.91 \mathrm{~d}$ & $1.56 \mathrm{c}$ \\
\hline CV (\%) & 2.91 & 9.13 & 5.19 & 9.18 & 5.94 & 9.14 & 6.60 & 6.93 & 6.85 \\
\hline
\end{tabular}

$\mathrm{T}_{1}: 100 \% \mathrm{STB}, \mathrm{T}_{2}: \mathrm{T}_{1}+25 \% \mathrm{~N}, \mathrm{~T}_{3}: \mathrm{T}_{1}+25 \% \mathrm{NP}, \mathrm{T}_{4}: \mathrm{T}_{1}+25 \% \mathrm{NK}, \mathrm{T}_{5}: \mathrm{T}_{1}+25 \% \mathrm{PK}, \mathrm{T}_{6}: \mathrm{T}_{1}+25 \% \mathrm{NPK}$, $\mathrm{T}_{7}: 75 \%$ of $\mathrm{T}_{1}, \mathrm{~T}_{8}$ : control treatment or native fertility. 
Table. 3. Yield and yield contributing characters of Mustard during the year 2018-2019

\begin{tabular}{|c|c|c|c|c|c|c|c|c|c|}
\hline \multirow{2}{*}{$\begin{array}{l}\text { Treat } \\
\text { ment }\end{array}$} & \multirow{2}{*}{$\begin{array}{c}\text { Plant } \\
\text { height } \\
(\mathrm{cm})\end{array}$} & \multirow{2}{*}{$\begin{array}{c}\text { No. of } \\
\text { branches }\end{array}$} & \multirow{2}{*}{$\begin{array}{l}\text { No. of } \\
\text { pods } \\
\text { plant }^{-1}\end{array}$} & \multirow{2}{*}{$\begin{array}{l}\text { Pod } \\
\text { length } \\
\text { (cm) }\end{array}$} & \multirow{2}{*}{$\begin{array}{l}\text { No. of } \\
\text { grains } \\
\text { pod }^{-1}\end{array}$} & \multirow{2}{*}{$\begin{array}{c}\text { Ineffective } \\
\text { pods } \\
\text { plant }^{-1}\end{array}$} & \multirow{2}{*}{$\begin{array}{c}1000- \\
\text { seed } \\
\text { weight }(\mathrm{g})\end{array}$} & $\begin{array}{l}\text { Seed } \\
\text { yield }\end{array}$ & yi \\
\hline & & & & & & & & \multicolumn{2}{|c|}{$\left(t \mathrm{ha}^{-1}\right)$} \\
\hline $\mathrm{T}_{1}$ & 950 & & & $4.65 b c$ & & & $3.32 \mathrm{~cd}$ & & \\
\hline $\mathrm{T}_{2}$ & & & & & & & & & \\
\hline $\mathrm{T}_{3}$ & & & & & & & & & \\
\hline $\mathrm{T}_{4}$ & & & & & & & & & \\
\hline $\mathrm{T}_{5}$ & 9 & & & 5 & & & 3.3 & bcd & $0 \mathrm{~b}$ \\
\hline $\mathrm{T}_{6}$ & & & & & & & & a & \\
\hline $\mathrm{T}_{7}$ & & & & & & & & $1.07 \mathrm{de}$ & \\
\hline & $c$ & & & & & & & $0.96 e$ & $0 \mathrm{c}$ \\
\hline $\mathrm{V}(\%)$ & 2.97 & & 6.88 & 6.98 & 8.23 & 7.32 & 6.98 & 9.11 & 7.96 \\
\hline
\end{tabular}

$\mathrm{T}_{1}: 100 \% \mathrm{STB}, \mathrm{T}_{2}: \mathrm{T}_{1}+25 \% \mathrm{~N}, \mathrm{~T}_{3}: \mathrm{T}_{1}+25 \% \mathrm{NP}, \mathrm{T}_{4}: \mathrm{T}_{1}+25 \% \mathrm{NK}, \mathrm{T}_{5}: \mathrm{T}_{1}+25 \% \mathrm{PK}, \mathrm{T}_{6}: \mathrm{T}_{1}+25 \% \mathrm{NPK}$, $\mathrm{T}_{7}: 75 \%$ of $\mathrm{T}_{1}, \mathrm{~T}_{8}$ : control treatment or native fertility.

\section{Boro}

The second component of the cropping pattern was Boro rice. The yield contributing characters and grain yield of Boro rice are presented in Table 4 and Table 5. Most of the yield contributing characters, straw and grain yield of Boro rice was significantly influenced by the different fertilizer treatments. Highest plant height was recorded from $\mathrm{T}_{6}$ treatment which was statistically at par with $\mathrm{T}_{2}, \mathrm{~T}_{3}, \mathrm{~T}_{4}$ and $\mathrm{T}_{5}$. In both the experimental years, 25\% NPK with 100\% STB gave highest no. of tiller hill ${ }^{-1}$, no of panicle hill ${ }^{-1}$, panicle length, grain and straw yield which was statistically similar with all other treatments except control plot or treatment. But numerically $\mathrm{T}_{6}$ treatment produced highest grain yield $\left(6.28\right.$ and $\left.6.34 \mathrm{t} \mathrm{ha}^{-1}\right)$ in both the consecutive years. Fertilizer has no significant effect on 1000-grain weight. Barman et al. (2019) also reported highest boro rice yield from 25\% extra NPK over 100\% STB dose in four crop based cropping pattern.

Table 4. Yield and yield contributing characters of Boro rice during the year 2018

\begin{tabular}{cccccccc}
\hline Treatments & $\begin{array}{c}\text { Plant } \\
\text { height } \\
\text { (cm) }\end{array}$ & $\begin{array}{c}\text { No. of } \\
\text { tiller } \\
\text { hill-1 }\end{array}$ & $\begin{array}{c}\text { No. of } \\
\text { panicle } \\
\text { hill-1 }\end{array}$ & $\begin{array}{c}\text { Panicle } \\
\text { length } \\
\text { (cm) }\end{array}$ & $\begin{array}{c}\text { 1000- } \\
\text { grain } \\
\text { wt. (g) }\end{array}$ & $\begin{array}{c}\text { Straw } \\
\text { yield }\end{array}$ & \multicolumn{2}{c}{$\begin{array}{c}\text { Grain } \\
\text { Yield ha }\end{array}$} \\
\hline $\mathrm{T}_{1}$ & $88.39 \mathrm{c}$ & $18.89 \mathrm{a}$ & $18.27 \mathrm{a}$ & $17.66 \mathrm{a}$ & 21.30 & $6.10 \mathrm{a}$ & $5.45 \mathrm{ab}$ \\
$\mathrm{T}_{2}$ & $90.07 \mathrm{ab}$ & $19.19 \mathrm{a}$ & $18.05 \mathrm{a}$ & $18.14 \mathrm{a}$ & 21.42 & $6.37 \mathrm{a}$ & $5.61 \mathrm{ab}$ \\
$\mathrm{T}_{3}$ & $91.12 \mathrm{ab}$ & $20.17 \mathrm{a}$ & $18.18 \mathrm{a}$ & $18.65 \mathrm{a}$ & 22.08 & $6.44 \mathrm{a}$ & $5.72 \mathrm{a}$ \\
$\mathrm{T}_{4}$ & $95.01 \mathrm{a}$ & $19.59 \mathrm{a}$ & $18.25 \mathrm{a}$ & $18.76 \mathrm{a}$ & 22.50 & $6.70 \mathrm{a}$ & $5.82 \mathrm{ab}$ \\
$\mathrm{T}_{5}$ & $95.42 \mathrm{a}$ & $19.95 \mathrm{a}$ & $18.35 \mathrm{a}$ & $19.02 \mathrm{a}$ & 22.89 & $6.89 \mathrm{a}$ & $5.73 \mathrm{ab}$ \\
$\mathrm{T}_{6}$ & $93.66 \mathrm{a}$ & $20.85 \mathrm{a}$ & $19.68 \mathrm{a}$ & $19.81 \mathrm{a}$ & 23.18 & $6.97 \mathrm{a}$ & $6.28 \mathrm{a}$ \\
$\mathrm{T}_{7}$ & $89.92 \mathrm{bc}$ & $20.08 \mathrm{a}$ & $17.25 \mathrm{a}$ & $18.31 \mathrm{a}$ & 21.42 & $5.27 \mathrm{a}$ & $5.35 \mathrm{ab}$ \\
$\mathrm{T}_{8}$ & $79.98 \mathrm{~d}$ & $15.15 \mathrm{~b}$ & $14.48 \mathrm{~b}$ & $16.45 \mathrm{~b}$ & 20.43 & $4.67 \mathrm{~b}$ & $4.79 \mathrm{~b}$ \\
\hline $\mathrm{CV}(\%)$ & 3.56 & 2.87 & 2.67 & 1.91 & 4.54 & 10.02 & 9.53 \\
\hline
\end{tabular}

$\mathrm{T}_{1}: 100 \%$ STB, $\mathrm{T}_{2}: \mathrm{T}_{1}+25 \% \mathrm{~N}, \mathrm{~T}_{3}: \mathrm{T}_{1}+25 \% \mathrm{NP}, \mathrm{T}_{4}: \mathrm{T}_{1}+25 \% \mathrm{NK}, \mathrm{T}_{5}: \mathrm{T}_{1}+25 \% \mathrm{PK}, \mathrm{T}_{6}: \mathrm{T}_{1}+25 \% \mathrm{NPK}$, $\mathrm{T}_{7}$ : $75 \%$ of $\mathrm{T}_{1}, \mathrm{~T}_{8}$ : control treatment or native fertility. 
Table 5. Yield and yield contributing characters of Boro rice during the year 2019

\begin{tabular}{cccccccc}
\hline Treatments & $\begin{array}{c}\text { Plant } \\
\text { height } \\
(\mathbf{c m})\end{array}$ & $\begin{array}{c}\text { No. of } \\
\text { tiller } \\
\text { hill }^{-1}\end{array}$ & $\begin{array}{c}\text { No. of } \\
\text { panicle } \\
\text { hill-1 }\end{array}$ & $\begin{array}{c}\text { Panicle } \\
\text { length } \\
\text { (cm) }\end{array}$ & $\begin{array}{c}\text { 1000- grain } \\
\text { wt. (g) }\end{array}$ & Straw yield & $\begin{array}{c}\text { Grain } \\
\text { Yield }\end{array}$ \\
\hline $\mathrm{T}_{1}$ & $85.33 \mathrm{c}$ & $19.74 \mathrm{a}$ & $19.22 \mathrm{a}$ & $19.44 \mathrm{a}$ & 22.36 & $6.13 \mathrm{a}$ & $5.43 \mathrm{ab}$ \\
$\mathrm{T}_{2}$ & $89.05 \mathrm{ab}$ & $19.66 \mathrm{a}$ & $19.02 \mathrm{a}$ & $19.42 \mathrm{a}$ & 23.22 & $6.12 \mathrm{a}$ & $5.52 \mathrm{ab}$ \\
$\mathrm{T}_{3}$ & $90.67 \mathrm{ab}$ & $20.04 \mathrm{a}$ & $19.06 \mathrm{a}$ & $19.74 \mathrm{a}$ & 22.04 & $6.18 \mathrm{a}$ & $5.81 \mathrm{a}$ \\
$\mathrm{T}_{4}$ & $93.65 \mathrm{a}$ & $19.73 \mathrm{a}$ & $19.11 \mathrm{a}$ & $19.76 \mathrm{a}$ & 21.36 & $6.34 \mathrm{a}$ & $5.76 \mathrm{ab}$ \\
$\mathrm{T}_{5}$ & $94.47 \mathrm{a}$ & $20.15 \mathrm{a}$ & $19.18 \mathrm{a}$ & $19.48 \mathrm{a}$ & 21.74 & $6.11 \mathrm{a}$ & $5.68 \mathrm{ab}$ \\
$\mathrm{T}_{6}$ & $93.82 \mathrm{a}$ & $20.22 \mathrm{a}$ & $19.48 \mathrm{a}$ & $19.68 \mathrm{a}$ & 23.18 & $6.13 \mathrm{a}$ & $6.34 \mathrm{a}$ \\
$\mathrm{T}_{7}$ & $86.74 \mathrm{bc}$ & $20.08 \mathrm{a}$ & $19.13 \mathrm{a}$ & $19.22 \mathrm{a}$ & 21.14 & $5.67 \mathrm{a}$ & $5.14 \mathrm{ab}$ \\
$\mathrm{T}_{8}$ & $76.82 \mathrm{~d}$ & $15.01 \mathrm{~b}$ & $14.44 \mathrm{~b}$ & $16.32 \mathrm{~b}$ & 20.73 & $4.32 \mathrm{~b}$ & $4.28 \mathrm{~b}$ \\
\hline $\mathrm{CV}(\%)$ & 3.36 & 2.64 & 2.45 & 2.67 & 5.12 & 9.86 & 8.68 \\
\hline
\end{tabular}

$\mathrm{T}_{1}: 100 \%$ STB, $\mathrm{T}_{2}: \mathrm{T}_{1}+25 \% \mathrm{~N}, \mathrm{~T}_{3}: \mathrm{T}_{1}+25 \% \mathrm{NP}, \mathrm{T}_{4}: \mathrm{T}_{1}+25 \% \mathrm{NK}, \mathrm{T}_{5}: \mathrm{T}_{1}+25 \% \mathrm{PK}, \mathrm{T}_{6}: \mathrm{T}_{1}+25 \% \mathrm{NPK}$, $\mathrm{T}_{7}$ : $75 \%$ of $\mathrm{T}_{1}, \mathrm{~T}_{8}$ : control treatment or native fertility.

\section{T. Aus Rice}

Data on yield and yield contributing characters of T. Aus rice was depicted in Table $6 \&$ \& . Both in 2018 and 2019, the tallest plant was recorded for $\mathrm{T}_{6}$ treatment but it was statistically similar with $T_{2}, T_{3}, T_{4}$ and $T_{5}$ treatment. No of tillers hill-1 ${ }^{-1}$, no of panicle hill-1 ${ }^{-1}$, panicle length and straw yield was statistically similar for $T_{1}, T_{2}, T_{3}, T_{4}$ and $T_{5}$ treatments but for $T_{8}$ treatment. 1000grain weight was not affected by fertilizer dose. During the experimental years of 2018 and 2019 , adding 25\% NPK over 100\% STB provided $44 \%$ and $50 \%$ higher yield, respectively than $75 \%$ of STB dose which was about $68 \%$ and $77 \%$ higher, respectively compared to native fertility. Barman et al. (2020) also reported 64\% higher yield from 25\% NPK+100\% STB dose than no fertilizer. An increase of N, P and K fertilizer doses from STB recommended doses significantly increased yield and yield contributing parameters of rice (Hasan et al., 2017).

Table 6. Yield and yield contributing characters of T. Aus rice during the year 2018

\begin{tabular}{|c|c|c|c|c|c|c|c|}
\hline \multirow{2}{*}{ Treatments } & \multirow{2}{*}{$\begin{array}{l}\text { Plant height } \\
\quad(\mathrm{cm})\end{array}$} & \multirow{2}{*}{$\begin{array}{c}\text { No. of tiller } \\
\text { hill }^{-1}\end{array}$} & \multirow{2}{*}{$\begin{array}{c}\text { No. of } \\
\text { panicle } \\
\text { hill-1 }\end{array}$} & \multirow{2}{*}{$\begin{array}{l}\text { Panicle } \\
\text { length } \\
\text { (cm) }\end{array}$} & \multirow{2}{*}{$\begin{array}{l}1000- \\
\text { grain wt. } \\
\text { (g) }\end{array}$} & $\begin{array}{l}\text { Straw } \\
\text { yield }\end{array}$ & $\begin{array}{l}\text { Grain } \\
\text { Yield }\end{array}$ \\
\hline & & & & & & \multicolumn{2}{|c|}{$\left(\right.$ t ha $\left.^{-1}\right)$} \\
\hline $\mathrm{T}_{1}$ & $82.13 c$ & $16.62 a$ & $16.18 \mathrm{a}$ & $16.44 a$ & 19.42 & $4.32 \mathrm{a}$ & $2.73 \mathrm{ab}$ \\
\hline $\mathrm{T}_{2}$ & $91.45 \mathrm{ab}$ & $16.64 a$ & $16.00 \mathrm{a}$ & $16.51 \mathrm{a}$ & 19.54 & $4.08 \mathrm{a}$ & $2.76 \mathrm{ab}$ \\
\hline $\mathrm{T}_{3}$ & $91.22 \mathrm{ab}$ & $16.98 a$ & $16.08 \mathrm{a}$ & $16.64 a$ & 19.62 & $4.04 a$ & $2.70 \mathrm{ab}$ \\
\hline $\mathrm{T}_{4}$ & $94.32 \mathrm{a}$ & $16.64 a$ & $16.17 \mathrm{a}$ & $16.72 \mathrm{a}$ & 19.71 & $4.48 \mathrm{a}$ & $2.74 a b$ \\
\hline $\mathrm{T}_{5}$ & $95.42 \mathrm{a}$ & $17.12 \mathrm{a}$ & $16.28 \mathrm{a}$ & $16.81 \mathrm{a}$ & 19.81 & $4.05 a$ & $2.80 \mathrm{ab}$ \\
\hline $\mathrm{T}_{6}$ & $93.87 a$ & $17.21 \mathrm{a}$ & $16.54 a$ & $16.92 a$ & 19.92 & $4.01 \mathrm{a}$ & $3.03 \mathrm{a}$ \\
\hline $\mathrm{T}_{7}$ & $87.19 b c$ & $17.00 \mathrm{a}$ & $16.18 \mathrm{a}$ & $16.31 \mathrm{a}$ & 19.38 & $3.74 a$ & $2.01 \mathrm{~b}$ \\
\hline $\mathrm{T}_{8}$ & $77.41 \mathrm{~d}$ & $15.02 b$ & $14.33 \mathrm{~b}$ & $14.22 \mathrm{~b}$ & 19.21 & $2.34 \mathrm{~b}$ & $1.20 \mathrm{c}$ \\
\hline $\mathrm{CV}(\%)$ & 3.87 & 2.89 & 3.55 & 1.86 & 4.57 & 10.23 & 8.11 \\
\hline
\end{tabular}

$\mathrm{T}_{1}: 100 \%$ STB, $\mathrm{T}_{2}: \mathrm{T}_{1}+25 \% \mathrm{~N}, \mathrm{~T}_{3}: \mathrm{T}_{1}+25 \% \mathrm{NP}, \mathrm{T}_{4}: \mathrm{T}_{1}+25 \% \mathrm{NK}, \mathrm{T}_{5}: \mathrm{T}_{1}+25 \% \mathrm{PK}, \mathrm{T}_{6}: \mathrm{T}_{1}+25 \% \mathrm{NPK}$, $\mathrm{T}_{7}: 75 \%$ of $\mathrm{T}_{1}, \mathrm{~T}_{8}$ : control treatment or native fertility. 
Table 7. Yield and yield contributing characters of T. Aus rice during the year 2019

\begin{tabular}{cccccccc}
\hline Treatments & $\begin{array}{c}\text { Plant } \\
\text { height }(\mathbf{c m})\end{array}$ & $\begin{array}{c}\text { No. of } \\
\text { tiller } \\
\text { hill-1 }^{-1}\end{array}$ & $\begin{array}{c}\text { No. of } \\
\text { panicle } \\
\text { hill-1 }\end{array}$ & $\begin{array}{c}\text { Panicle } \\
\text { length } \\
(\mathbf{c m})\end{array}$ & $\begin{array}{c}1000- \\
\text { grain } \\
\text { wt. (g) }\end{array}$ & $\begin{array}{c}\text { Straw } \\
\text { yield }\end{array}$ & $\begin{array}{c}\text { Grain } \\
\text { Yield }\end{array}$ \\
\hline $\mathrm{T}_{1}$ & $82.00 \mathrm{c}$ & $15.44 \mathrm{~h} \mathbf{h}^{-1}$ ) & $15.14 \mathrm{a}$ & $15.62 \mathrm{a}$ & 19.42 & $4.32 \mathrm{a}$ & $2.71 \mathrm{ab}$ \\
$\mathrm{T}_{2}$ & $90.12 \mathrm{ab}$ & $15.55 \mathrm{a}$ & $15.12 \mathrm{a}$ & $15.65 \mathrm{a}$ & 19.35 & $4.13 \mathrm{a}$ & $2.73 \mathrm{ab}$ \\
$\mathrm{T}_{3}$ & $90.08 \mathrm{ab}$ & $16.11 \mathrm{a}$ & $15.07 \mathrm{a}$ & $15.96 \mathrm{a}$ & 19.26 & $4.06 \mathrm{a}$ & $2.75 \mathrm{ab}$ \\
$\mathrm{T}_{4}$ & $93.22 \mathrm{a}$ & $15.77 \mathrm{a}$ & $15.14 \mathrm{a}$ & $15.97 \mathrm{a}$ & 19.47 & $4.47 \mathrm{a}$ & $2.77 \mathrm{ab}$ \\
$\mathrm{T}_{5}$ & $94.31 \mathrm{a}$ & $16.22 \mathrm{a}$ & $15.18 \mathrm{a}$ & $15.68 \mathrm{a}$ & 19.58 & $4.08 \mathrm{a}$ & $2.88 \mathrm{ab}$ \\
$\mathrm{T}_{6}$ & $92.71 \mathrm{a}$ & $16.11 \mathrm{a}$ & $15.33 \mathrm{a}$ & $15.89 \mathrm{a}$ & 19.39 & $4.09 \mathrm{a}$ & $3.19 \mathrm{a}$ \\
$\mathrm{T}_{7}$ & $86.12 \mathrm{bc}$ & $15.98 \mathrm{a}$ & $15.02 \mathrm{a}$ & $15.33 \mathrm{a}$ & 19.31 & $3.71 \mathrm{a}$ & $2.21 \mathrm{~b}$ \\
$\mathrm{~T}_{8}$ & $76.31 \mathrm{~d}$ & $14.11 \mathrm{~b}$ & $13.21 \mathrm{~b}$ & $13.66 \mathrm{~b}$ & 19.31 & $2.40 \mathrm{~b}$ & $1.10 \mathrm{c}$ \\
\hline $\mathrm{CV}(\%)$ & 4.12 & 5.03 & 3.18 & 2.34 & 4.55 & 8.98 & 9.20 \\
\hline
\end{tabular}

$\mathrm{T}_{1}: 100 \%$ STB, $\mathrm{T}_{2}: \mathrm{T}_{1}+25 \% \mathrm{~N}, \mathrm{~T}_{3}: \mathrm{T}_{1}+25 \% \mathrm{NP}, \mathrm{T}_{4}: \mathrm{T}_{1}+25 \% \mathrm{NK}, \mathrm{T}_{5}: \mathrm{T}_{1}+25 \% \mathrm{PK}, \mathrm{T}_{6}: \mathrm{T}_{1}+25 \% \mathrm{NPK}$, $\mathrm{T}_{7}$ : $75 \%$ of $\mathrm{T}_{1}, \mathrm{~T}_{8}$ : control treatment or native fertility.

\section{T. Aman}

The forth component of the cropping pattern was T. Aman rice. Table 8 and Table 9 represented the experimental data related to yield and yield contributing characters of T. Aman rice. $\mathrm{T}_{6}$ treatment produced the tallest plant which was statistically similar with all other treatment except $T_{7}$ and $T_{8}$. Control treatment produced the lowest no. of tillers hill ${ }^{-1}$ while other treatments showed no significant variations in both the consecutive years. There was no significant impact of treatments on panicle length and 1000-grain weight. Highest straw yield was recorded from $T_{6}$ and lowest from $T_{8}$. During the two consecutive experimental seasons of 2018 and 2019, about 93\% and 88\% higher yield, respectively was recorded from $\mathrm{T}_{6}$ treatment than control but it was statistically similar with $100 \%$ STB dose and other treatments. Ali et al. (2009) observed maximum grain yield from soil test value for HYG while Saha et al. (2016) reported higher yield from $20 \%$ more from the STB dose in potato-maize-T. Aman cropping pattern of AEZ-3.

Table 8. Yield and yield contributing characters of T. Aman rice during the year 2018

\begin{tabular}{|c|c|c|c|c|c|c|c|}
\hline \multirow{2}{*}{ Treatments } & \multirow{2}{*}{$\begin{array}{l}\text { Plant height } \\
\text { (cm) }\end{array}$} & \multirow{2}{*}{$\begin{array}{c}\text { No. of } \\
\text { tiller } \\
\text { hill-1 }^{-1}\end{array}$} & \multirow{2}{*}{$\begin{array}{c}\text { No. of } \\
\text { panicle } \\
\text { hill }^{-1}\end{array}$} & \multirow{2}{*}{$\begin{array}{c}\text { Panicle } \\
\text { length } \\
\text { (cm) }\end{array}$} & \multirow{2}{*}{$\begin{array}{c}1000- \\
\text { grain } \\
\text { wt. (g) }\end{array}$} & $\begin{array}{c}\text { Straw } \\
\text { yield }\end{array}$ & $\begin{array}{c}\text { Grain } \\
\text { yield }\end{array}$ \\
\hline & & & & & & \multicolumn{2}{|c|}{$\left(\mathrm{t} \mathrm{ha}^{-1}\right)$} \\
\hline$T_{1}$ & $96.42 \mathrm{ab}$ & $15.44 a$ & $15.43 b$ & 18.41 & 21.41 & $5.43 \mathrm{ab}$ & $3.32 \mathrm{a}$ \\
\hline $\mathrm{T}_{2}$ & $96.51 \mathrm{ab}$ & $17.53 \mathrm{a}$ & $16.52 \mathrm{ab}$ & 18.55 & 21.53 & $5.53 \mathrm{abc}$ & $3.45 a$ \\
\hline $\mathrm{T}_{3}$ & $97.64 a b$ & $17.61 \mathrm{a}$ & $16.61 \mathrm{ab}$ & 18.66 & 21.62 & $5.61 \mathrm{abc}$ & $3.55 a$ \\
\hline $\mathrm{T}_{4}$ & $97.72 \mathrm{ab}$ & $16.72 \mathrm{a}$ & $16.72 \mathrm{ab}$ & 18.73 & 21.73 & $4.71 b c$ & $3.65 a$ \\
\hline $\mathrm{T}_{5}$ & $98.82 \mathrm{a}$ & $17.81 \mathrm{a}$ & $16.81 \mathrm{ab}$ & 18.84 & 21.83 & $5.81 \mathrm{ab}$ & $3.75 a$ \\
\hline $\mathrm{T}_{6}$ & $98.94 a$ & $17.91 \mathrm{a}$ & $16.91 \mathrm{a}$ & 18.95 & 21.93 & $5.91 \mathrm{a}$ & $4.08 \mathrm{a}$ \\
\hline $\mathrm{T}_{7}$ & $94.32 b$ & $16.33 a$ & $15.33 \mathrm{ab}$ & 18.32 & 21.31 & $4.31 b c$ & $3.23 a$ \\
\hline $\mathrm{T}_{8}$ & $72.21 \mathrm{c}$ & $9.23 b$ & $9.23 c$ & 18.20 & 21.21 & $3.23 c$ & $2.11 \mathrm{~b}$ \\
\hline CV (\%) & 2.88 & 4.77 & 4.75 & 5.12 & 4.11 & 10.82 & 7.32 \\
\hline
\end{tabular}

$\mathrm{T}_{1}: 100 \%$ STB, $\mathrm{T}_{2}: \mathrm{T}_{1}+25 \% \mathrm{~N}, \mathrm{~T}_{3}: \mathrm{T}_{1}+25 \% \mathrm{NP}, \mathrm{T}_{4}: \mathrm{T}_{1}+25 \% \mathrm{NK}, \mathrm{T}_{5}: \mathrm{T}_{1}+25 \% \mathrm{PK}, \mathrm{T}_{6}: \mathrm{T}_{1}+25 \% \mathrm{NPK}$,

$\mathrm{T}_{7}$ : $75 \%$ of $\mathrm{T}_{1}, \mathrm{~T}_{8}$ : control treatment or native fertility. 
Table 9. Yield and yield contributing characters of T. Aman rice during 2019

\begin{tabular}{cccccccc}
\hline Treatments & $\begin{array}{c}\text { Plant height } \\
(\mathbf{c m})\end{array}$ & $\begin{array}{c}\text { No. of } \\
\text { tiller } \\
\text { hill-1 }\end{array}$ & $\begin{array}{c}\text { No. of } \\
\text { panicle } \\
\text { hill-1 }\end{array}$ & $\begin{array}{c}\text { Panicle } \\
\text { length } \\
(\mathbf{c m})\end{array}$ & $\begin{array}{c}\mathbf{1 0 0 0} \\
\text { grain } \\
\text { wt. (g) }\end{array}$ & $\begin{array}{c}\text { Straw } \\
\text { yield }\end{array}$ & $\begin{array}{c}\text { Grain } \\
\text { yield }\end{array}$ \\
\hline $\mathrm{T}_{1}$ & $95.54 \mathrm{t} \mathbf{h a}^{-\mathbf{1}}$ ) \\
$\mathrm{T}_{2}$ & $95.55 \mathrm{ab}$ & $15.84 \mathrm{a}$ & $15.13 \mathrm{~b}$ & 18.44 & 21.04 & $5.14 \mathrm{ab}$ & $3.53 \mathrm{a}$ \\
$\mathrm{T}_{3}$ & $96.56 \mathrm{ab}$ & $16.75 \mathrm{a}$ & $16.24 \mathrm{ab}$ & 18.05 & 21.55 & $5.05 \mathrm{abc}$ & $3.54 \mathrm{a}$ \\
$\mathrm{T}_{4}$ & $96.47 \mathrm{ab}$ & $15.97 \mathrm{a}$ & $15.46 \mathrm{ab}$ & 18.96 & 21.26 & $5.06 \mathrm{abc}$ & $3.65 \mathrm{a}$ \\
$\mathrm{T}_{5}$ & $97.58 \mathrm{a}$ & $16.02 \mathrm{a}$ & $15.87 \mathrm{ab}$ & 18.38 & 21.58 & $5.78 \mathrm{ab}$ & $3.57 \mathrm{a}$ \\
$\mathrm{T}_{6}$ & $97.59 \mathrm{a}$ & $16.11 \mathrm{a}$ & $16.09 \mathrm{a}$ & 18.19 & 21.59 & $5.89 \mathrm{a}$ & $3.98 \mathrm{a}$ \\
$\mathrm{T}_{7}$ & $93.63 \mathrm{~b}$ & $15.74 \mathrm{a}$ & $15.12 \mathrm{ab}$ & 18.72 & 21.33 & $4.52 \mathrm{bc}$ & $3.22 \mathrm{a}$ \\
$\mathrm{T}_{8}$ & $71.42 \mathrm{c}$ & $9.63 \mathrm{~b}$ & $9.02 \mathrm{c}$ & 18.51 & 21.22 & $3.81 \mathrm{c}$ & $2.08 \mathrm{~b}$ \\
\hline $\mathrm{CV}(\%)$ & 3.11 & 4.98 & 5.21 & 5.55 & 4.11 & 9.22 & 8.98 \\
\hline
\end{tabular}

$\mathrm{T}_{1}: 100 \%$ STB, $\mathrm{T}_{2}: \mathrm{T}_{1}+25 \% \mathrm{~N}, \mathrm{~T}_{3}: \mathrm{T}_{1}+25 \% \mathrm{NP}, \mathrm{T}_{4}: \mathrm{T}_{1}+25 \% \mathrm{NK}, \mathrm{T}_{5}: \mathrm{T}_{1}+25 \% \mathrm{PK}, \mathrm{T}_{6}: \mathrm{T}_{1}+25 \% \mathrm{NPK}$, $\mathrm{T}_{7}$ : $75 \%$ of $\mathrm{T}_{1}, \mathrm{~T}_{8}$ : control treatment or native fertility.

\section{Rice equivalent yield}

Productivity of different crops on the rice based cropping system was determined by Rice Equivalent Yield (REY) which was calculated from yield of component crops. Average yield of component crops and rice equivalent yield of mustard was depicted in Table 10. From the results of two years research on four crop based cropping pattern, it was observed that the highest REY (3.34 $\left.\mathrm{t} \mathrm{ha}^{-1}\right)$ was recorded from $\mathrm{T}_{6}\left(25 \%\right.$ extra NPK over $100 \%$ STB dose) and lowest from $\mathrm{T}_{8}$.

REY of mustard was $46 \%$ higher than 100\% STB dose when 25\% more NPK was added to it. Barman et al. (2020) also documented higher rice equivalent yield of mustard from 25\% NPK+ $100 \%$ STB dose. Mondal et al. (2015) also claimed of having 49 to $67 \%$ higher productivity from the intensified land use system under four cropped based cropping pattern. Inclusion of mustard in rice based cropping pattern increase REY about 45.3-51.6\% (Hossain et al., 2014). Naher et al. (2016) and Hossain et al. (2014) opined that short duration varieties and improved four crop based cropping pattern increases total productivity and profitability over farmers existing pattern.

Table 10. Rice equivalent yield of Mustard-Boro-T. Aus-T. Aman

\begin{tabular}{cccccc}
\hline \multirow{2}{*}{ Treatments } & \multicolumn{3}{c}{ Average yield of crops in the pattern } & \multirow{2}{*}{$\begin{array}{c}\text { Rice equivalent yield } \\
\text { mustard in the pattern }\end{array}$} \\
\cline { 2 - 5 } & Mustard & Boro & T.aus & T.aman & 2.26 \\
\hline $\mathrm{T}_{1}$ & 1.13 & 5.44 & 2.72 & 3.43 & 2.48 \\
$\mathrm{~T}_{2}$ & 1.24 & 5.57 & 2.75 & 3.50 & 3.06 \\
$\mathrm{~T}_{3}$ & 1.53 & 5.77 & 2.73 & 3.60 & 2.64 \\
$\mathrm{~T}_{4}$ & 1.32 & 5.79 & 2.76 & 3.56 & 2.58 \\
$\mathrm{~T}_{5}$ & 1.29 & 5.71 & 2.84 & 3.66 & 3.34 \\
$\mathrm{~T}_{6}$ & 1.67 & 6.32 & 3.11 & 4.03 & 2.08 \\
$\mathrm{~T}_{7}$ & 1.04 & 5.25 & 2.11 & 3.23 & 1.88 \\
$\mathrm{~T}_{8}$ & 0.94 & 4.53 & 1.15 & 2.10 & \\
\hline
\end{tabular}

Price $: 1 \mathrm{~kg}$ mustard $=\mathrm{Tk} .50,1 \mathrm{~kg}$ rice $=\mathrm{Tk} .25$

$\mathrm{T}_{1}: 100 \% \mathrm{STB}, \mathrm{T}_{2}: \mathrm{T}_{1}+25 \% \mathrm{~N}, \mathrm{~T}_{3}: \mathrm{T}_{1}+25 \% \mathrm{NP}, \mathrm{T}_{4}: \mathrm{T}_{1}+25 \% \mathrm{NK}, \mathrm{T}_{5}: \mathrm{T}_{1}+25 \% \mathrm{PK}, \mathrm{T}_{6}: \mathrm{T}_{1}+25 \% \mathrm{NPK}$, $\mathrm{T}_{7}$ : $75 \%$ of $\mathrm{T}_{1}, \mathrm{~T}_{8}$ : control treatment or native fertility. 


\section{Economic analysis}

Cost and return analysis was done on the basis of prevailing market price of the commodities. Economics analysis of the cropping pattern Mustard-Boro-T.Aus-T.Aman was delineated in Table 11. It was observed that highest gross return, total variable cost, gross margin and marginal value product was obtained from $25 \%$ extra NPK over 100\% STB dose. The maximum marginal benefit cost ratio was also gained from $\mathrm{T}_{6}$ treatment. Although the total variable cost is higher for $\mathrm{T}_{6}$ but its output value surpassed other fertilizer packages. That's why $25 \%$ more NPK with 100\% STB dose was the most economically profitable fertilizer package for Mustard-BoroT.Aus -T.Aman cropping pattern under AEZ-11. Hossain et al. (2014) found highest MBCR from Mustard-Boro-T.Aus-T.Aman. Thus four crop based cropping pattern would play a vital role to ensure food security of the country in upcoming days (Mondal et al., 2014) and also improve socio-economic condition of farmers.

Table 11.Economic analysis of the cropping pattern Mustard- Boro-T. Aus-T. Aman

\begin{tabular}{cccccc}
\hline Treatments & $\begin{array}{c}\text { Gross return of } \\
\text { the system }\end{array}$ & $\begin{array}{c}\text { Total variable } \\
\text { cost }\end{array}$ & Gross margin & $\begin{array}{c}\text { Marginal product } \\
\text { value (MVP) }\end{array}$ & $\begin{array}{c}\text { Marginal benefit } \\
\text { cost ratio } \\
\text { (MBCR) }\end{array}$ \\
\cline { 2 - 4 } & \multicolumn{2}{c}{ (Tk./ha) } & 104750 & 2.57 \\
\hline $\mathrm{T}_{1}$ & 346250 & 40708 & 305542 & 116000 & 2.75 \\
$\mathrm{~T}_{2}$ & 357500 & 42231 & 315269 & 137500 & 3.18 \\
$\mathrm{~T}_{3}$ & 379000 & 43252 & 335748 & 127250 & 2.98 \\
$\mathrm{~T}_{4}$ & 368750 & 42682 & 326068 & 128250 & 3.04 \\
$\mathrm{~T}_{5}$ & 369750 & 42180 & 327570 & 178500 & 4.08 \\
$\mathrm{~T}_{6}$ & 420000 & 43703 & 376297 & 75250 & 2.46 \\
$\mathrm{~T}_{7}$ & 316750 & 30531 & 286219 & - & - \\
$\mathrm{T}_{8}$ & 241500 & 0 & 241500 & & \\
\hline
\end{tabular}

Urea $=16 \mathrm{Tk} \mathrm{kg}^{-1}, \mathrm{TSP}=25 \mathrm{Tk} \mathrm{kg}^{-1}, \mathrm{MoP}=15 \mathrm{Tk} \mathrm{kg}^{-1}$, Gypsum $=12 \mathrm{Tk} \mathrm{kg}^{-1}$, Zinc sulphate $=200 \mathrm{Tk} \mathrm{kg}^{-1}$

$\mathrm{T}_{1}: 100 \% \mathrm{STB}, \mathrm{T}_{2}: \mathrm{T}_{1}+25 \% \mathrm{~N}, \mathrm{~T}_{3}: \mathrm{T}_{1}+25 \% \mathrm{NP}, \mathrm{T}_{4}: \mathrm{T}_{1}+25 \% \mathrm{NK}, \mathrm{T}_{5}: \mathrm{T}_{1}+25 \% \mathrm{PK}, \mathrm{T}_{6}: \mathrm{T}_{1}+25 \% \mathrm{NPK}$, $\mathrm{T}_{7}: 75 \%$ of $\mathrm{T}_{1}, \mathrm{~T}_{8}$ : control treatment or native fertility.

\section{Conclusion}

Considering the system productivity, and cost and return analysis of the experiment, it may be concluded that $25 \%$ extra NPK with $100 \%$ STB dose is agronomically feasible and economically profitable for Mustard-Boro-T.Aus-T.Aman cropping pattern in AEZ-11. So, this fertilizer dose may be followed for a remunerative and productive rice based Mustard-Boro-T. Aus-T. Aman cropping system under Jashore region. Further experimentation will be required under different AEZs' to find out suitable region specific fertilizer packages for this pattern.

\section{References}

Ali, M.R., D.J. Costa, M.A. Sayed, M.A.H. Khanand J.A. Abedin. 2009. Development of fertilizer recommendation for the cropping pattern Potato-Boro-T. Aman in irrigated medium highland condition under AEZ -9. Bangladesh J. Agril. Res. 34(1): 41-49.

Aziz I, T. Mahmood, K.R. Islam and E. Ashraf. 2013. Estimation of cropping system impacts on C and $\mathrm{N}$ status of soil. Int. J. Agric. Appl. Sci. 5(1): 1-7.

Barman, A., M.M. Masud, S. Akhter, M.A. Hossain and S. Shome. 2020. Development of fertilizer recommendation of four crops grown under the cropping pattern: Mustard-Boro- $\mathrm{T}$. Aus- T. Aman in AEZ-8. Bangladesh J. Agril. Res. 45(2): 113-123. 
Barman, A., S. Shome, M. J. Alam, S. Akhter and M.A. Hossain. 2019. Fertilizer recommendation for four crop based cropping pattern: Potato- Boro- T. Aus-T. Aman under AEZ-11. Bangladesh Agron. J. 22(1): 71-78.

BBS. 2019. Statistical Yearbook of Bangladesh. 39th edition. Statistics Division, Ministry of Planning, Govt. of the People's Republic of Bangladesh. p.47.

Dobermann, A., R. Nelson, D. Beever, D. Bergvinson, E. Crowley, G. Denning, K. Giller, J. d'Arros Hughes, M. Jahn, J. Lynam, W. Masters, R. Naylor, G. Neath, I. Onyido, T. Remington, I. Wrightand and F. Zhang. 2013. Solutions for sustainable agriculture and food systems. Technical report for the post-2015 development agenda. Sustainable Development Solutions Network, New York.

Hasan, A.B.M.M., S.M. Shamsuzzaman, H.A.K. Chowdhury, S.M. Rasel, N.H. Khan, M.E. Rahman, M. Mehnaz and A.W. Samsuri. 2017. Effect of different fertilizer packages on the yield and yield attributes on BRRI dhan47 in saline areas. Bangladesh J. Bot. 46(1): 467-472.

Hossain, I., M.R.I. Mondal, M.J. Islam, M.A. Aziz, A.S.M.M.R. Khan and F. Begum. 2014. Four crop based cropping pattern studies for increasing cropping intensity and productivity in Rajshahi region of Bangladesh. Bangladesh Agron. J. 17(2): 55-60.

Islam, M.A., M.J. Islam, M.A. Ali, A.S.M.M.R. Khan, M.F. Hossain and M. Moniruzzaman. 2018. Transforming triple cropping system to four crops pattern: An approach of enhancing system productivity through intensifying land use system in Bangladesh. Int. J. Agon. DOI: pageshttps://doi.org/10.1155/2018/7149835.

Mondal, R.I., F. Begum, A. Aziz and S.H. Sharif. 2015. Crop sequences for increasing cropping intensity and productivity. SAARC J. Agril. 13(1): 135- 147.

Naher Q., M.R. Amin., M.R. Islam M.A. Ali, A.K. Choudhury, M.K. Hasan and A.S.M.M.R. Khan. 2016. Location specific climate resilient agricultural technologies in Bangladesh. OFRD (OnFarm Research Division), BARI, Bangladesh. pp.37-38.

Parvin, N., A. Khatun, M.K. Quais and M. Nasim. 2017. Cropping Pattern, Intensity and Diversity in Dhaka Region. Bangladesh Rice J. 21(2): 123-141.

Saha, P.K., F. Rahman, M. Akter, R. Islam, A.T.M.S. Hossain and M.G. Ali. 2016. Integrated nutrient management for Potato-Maize-T. Aman rice cropping pattern in Bangladesh. Rice J. 20(1): 51-58.

Sultana, J., M.N.A. Siddique and M.R. Abdullah. 2015. Fertilizer recommendation for Agriculture: practice, practicalities and adaptation in Bangladesh and Netherlands. Int. J. Bus. Manag. Soc. Res. 1(1): 21-40. 